\title{
ERRATUM
}

\section{Frequent users of an inner-city emergency department - ERRATUM}

doi:10.2310/8000.2012.120670

In the original publication of "Frequent users of an inner-city emergency department," an additional author, Karen Anne Sutherland, was not listed. The correct author listing of this article should be: Jill Geurts, Wes Palatnick, Trevor Strome, Karen Anne Sutherland, and Erin Weldon.

The CFEM editors regret this error.

\section{REFERENCE}

Geurts J, Palatnick W, Strome T, Sutherland KA, Weldon E. Frequent users of an inner-city emergency department. CFEM 2012;14(5):306-13. 